\title{
Network-based Collaborative Navigation in GPS-Denied Environment
}

\author{
Jong Ki Lee ${ }^{1,2}$, Dorota A. Grejner-Brzezinska ${ }^{1}$ and Charles Toth ${ }^{1,3}$ \\ ${ }^{1}$ (Satellite Positioning and Inertial Navigation [SPIN] Laboratory, Department of \\ Civil and Environmental Engineering and Geodetic Science, The Ohio State University, \\ Ohio, USA) \\ ${ }^{2}$ (Division of Geodetic Science, School of Earth Science, The Ohio State University, \\ Ohio, USA) \\ ${ }^{3}$ (Center for Mapping, The Ohio State University, Ohio, USA) \\ (E-mail: jk12608@gmail.com)
}

Global Positioning System (GPS) has been used as a primary source of navigation in land and airborne applications. However, challenging environments cause GPS signal blockage or degradation, and prevent reliable and seamless positioning and navigation using GPS only. Therefore, multi-sensor based navigation systems have been developed to overcome the limitations of GPS by adding some forms of augmentation. The next step towards assured robust navigation is to combine information from multiple ground-users, to further improve the chance of obtaining reliable navigation and positioning information. Collaborative (or cooperative) navigation can improve the individual navigation solution in terms of both accuracy and coverage, and may reduce the system's design cost, as equipping all users with high performance multi-sensor positioning systems is not cost effective. Generally, 'Collaborative Navigation' uses inter-nodal range measurements between platforms (users) to strengthen the navigation solution. In the collaborative navigation approach, the inter-nodal distance vectors from the known or more accurate positions to the unknown locations can be established. Therefore, the collaborative navigation technique has the advantage in that errors at the user's position can be compensated by other known (or more accurate) positions of other platforms, and may result in the improvement of the navigation solutions for the entire group of users. In this paper, three statistical network-based collaborative navigation algorithms, the Restricted Least-Squares Solution (RLESS), the Stochastic Constrained Least-Squares Solution (SCLESS) and the Best Linear Minimum Partial Bias Estimation (BLIMPBE) are proposed and compared to the Kalman filter. The proposed statistical collaborative navigation algorithms for network solution show better performance than the Kalman filter.

\section{KEY WORDS}
1. Collaborative Navigation.
2. Network-based Estimation.
3. RLESS, SCLESS, BLIMPBE. 4. Kalman Filter.

First published online: 23 March 2012.

1. INTRODUCTION. Global Positioning System (GPS) is the most widely used positioning and navigation technology. Differential GPS (DGPS) offers centimetre-level positioning accuracy for static observations and sub-decimetre 


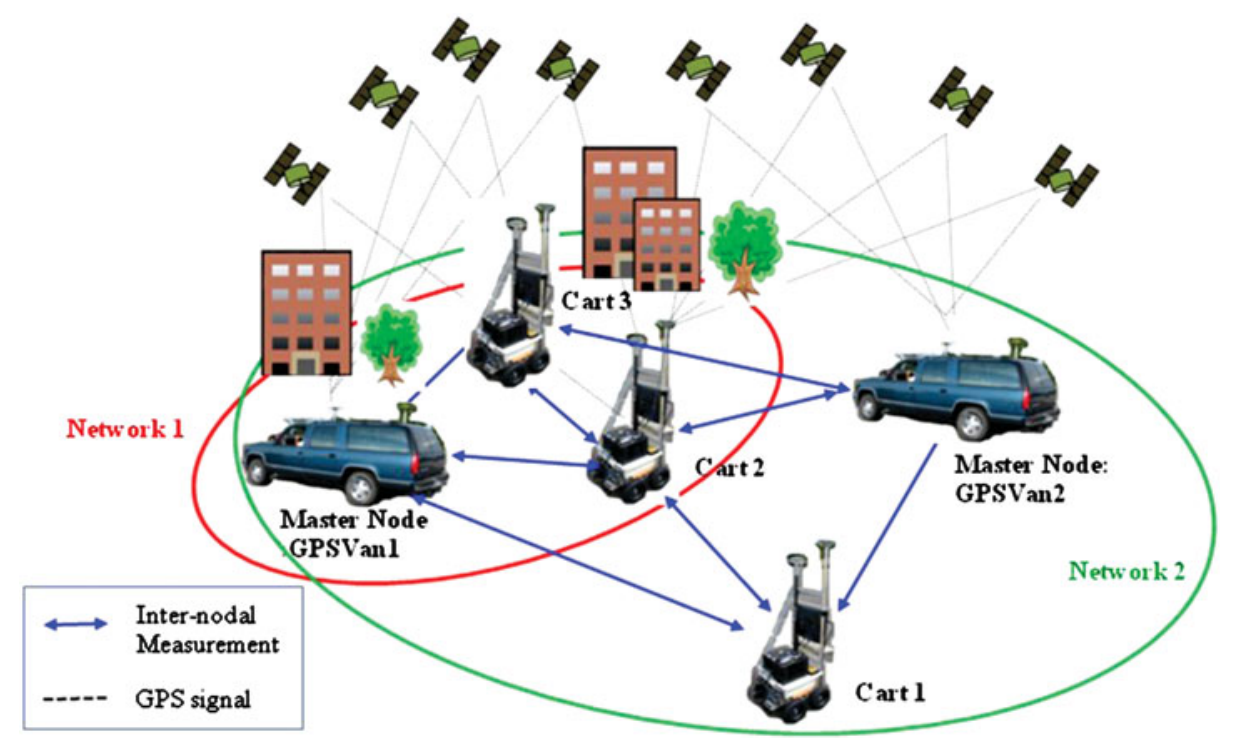

Figure 1. Collaborative navigation concept.

accuracy for kinematic systems. However, GPS is a line-of-sight system and is, therefore, unusable or severely limited by signal availability in GPS-challenged environments. GPS is also subject to intentional (jamming) and unintentional RF interference. To overcome these limitations, many navigation technologies based mainly on the concept of multi-sensor integration have been proposed and tested (Mirabadi et al., 2003; Sharaf and Noureldin, 2007; Grejner-Brzezinska et al., 2008; Lee and Jekeli, 2011).

In the concept of Collaborative Navigation (CN), each vehicle (also referred to as node hereafter) in the network share information (e.g. GPS code pseudo-ranges, carrier-phase pseudo-ranges or its 3-Dimensional (3D) coordinates, and the internodal distance measurements with other nodes) to improve the overall performance of a group of vehicles. It is assumed that each node of the network can measure the 3D relative position by various inter-nodal ranging techniques such as laser ranging, microwave ranging and vision sensors. The $\mathrm{CN}$ can improve the accuracy of a singleplatform navigation solution, increase the integrity of its solution, and detect possible collision causes of the vehicle. Figure 1 illustrates the network-based collaborative navigation concept where sub-networks of nodes navigating jointly could be created ad hoc, as indicated by the circles; it should be noted that some nodes (users) may be parts of different sub-networks. In a whole network, the selection of a sub-network of nodes is a critical issue, as in the case of a large number of users in the entire network, computational and communication loads may not allow for the entire network to be treated as one entity (Grejner-Brzezinska et al., 2009). Conceptually, the sub-networks can consist of nodes of equal hierarchy or may contain a master (anchor) node that will normally have a better set of sensors and will be collecting measurements from all other nodes to perform collaborative navigation.

Various collaborative navigation technologies based on the multi-sensor concept have been discussed in many literatures. (Sanderson, 1997) employed the range 
measurement in the centralized linear Kalman filter framework. (Fox et al., 1999) proposed collaborative mobile robot localization based on a probabilistic navigation algorithm. (Berefelt et al., 2004) showed that the relative vector method was better than the other two methods (virtual satellite and shared pseudorange) to improve the GPS/ INS performance in urban navigation. (Panzieri et al., 2005) used a bank of interlaced Kalman filters to incorporate ranges into the states of the filter. (Brown and Nordlie, 2006) designated the master nodes to improve the navigation performance of the entire network. (Kaba and Wu, 2009) developed the distributed multi-sensor fusion to bind the position errors using GPS, RF beacons, or anchor nodes for the collaborative navigation system. (Grejner-Brzezinska et al., 2009) employed the bank of extended Kalman filters to integrate the inter-nodal range measurements with other sensor.

Collaborative navigation has advantage over the single vehicle navigation because the position errors of some nodes can be compensated by known (or more accurate) coordinates of other nodes, which improves the overall navigation results of the group of users navigating together. It should be noted that previous studies of the networkbased approach use primarily the distance-dependent weighting or the empirical covariance, thus, the accuracy and reliability of a single user or a group of users may not be significantly improved. In this paper, three statistical network-based collaborative navigation algorithms, namely the Stochastic Constrained LeastSquares Solution (SCLESS), the Best Linear Minimum Partial Bias Estimation (BLIMPBE), and the Restricted Least-Squares Solution (RLESS) are proposed and compared to the Kalman filter, based on the field data collected by a network of five kinematic platforms; a GPS Van, a mobile cart and three GPS stations.

\section{NETWORK-BASED COLLABORATIVE NAVIGATION}

ALGORITHM. The relative component of the inter-nodal vector between nodes $i$ and $j$ is given as:

$$
d_{i j}=x_{i}-x_{j},
$$

where:

$d_{i j}$ is a single component of the actual distance measurements between two nodes. $x_{i}$ and $x_{j}$ are the position coordinates of each node.

The inter-nodal output measurement (i.e., sensor output) is simply modeled as equation (2) with additive noise $v$ :

$$
y_{i j}=d_{i j}+v
$$

where $v$ is assumed to have Gaussian distribution $\left(v \sim N\left(0, R_{v}\right)\right.$, where $R_{v}$ is the noise covariance matrix.

The three-dimensional measurement model for the $n$-node network environment is given as:

$$
\underset{[3 m \times 1]}{\vec{y}}=\underset{[3 m \times 3 n]}{A} \cdot \underset{[3 n \times 1]}{\vec{x}}+\underset{[3 m \times 1]}{\vec{v}}
$$

where:

$\vec{y}$ is the vector of $\mathrm{x}, \mathrm{y}, \mathrm{z}$ difference of inter-nodal distance measurements.

$m$ is the number of measurements. 


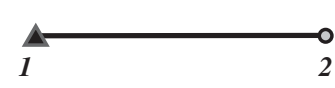

(a) two nodes

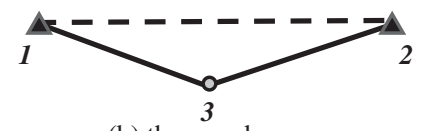

(b) three nodes

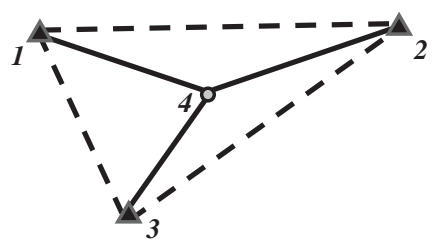

(c) four nodes

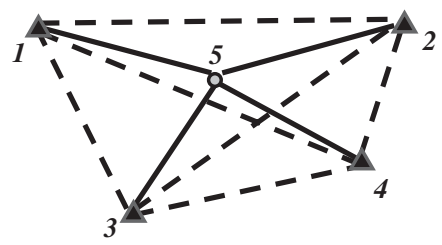

(d) five nodes

Figure 2. The geometry of networks studied here as a function of a number of nodes: $\boldsymbol{\Delta}$ : reference node, $\mathbf{O}$ : user node.

$\vec{x}$ is the vector of position in $\mathrm{x}, \mathrm{y}, \mathrm{z}$ for all nodes in the network.

$A$ is the design matrix that linearly relates the node variables through the inter-nodal measurements, and $\vec{v}$ is the additive noise vector.

Since the design matrix $A$ shows the relationship of the relative inter-nodal measurements for pairs of nodes, it can be written as a composite matrix of identity matrices. The dimension of matrix $A$ is $3 m$ by $3 n$ for the three dimensional coordinates. In the examples shown here, it is assumed that there is only one user node and other nodes are all reference (or higher order) nodes in a network. Thus, for instance, if the number of nodes is three, there are two inter-nodal distance measurements between the user node and two reference nodes $d_{31}$ and $d_{32}$ in Figure $2 \mathrm{~b}$, then the matrix $A$ is given as follows:

$$
A=\left[\begin{array}{ccc}
-I_{1} & 0 & I_{3} \\
0 & -I_{1} & I_{3}
\end{array}\right]
$$

where each $I$ is a 3 by 3 identity matrix for the $3 \mathrm{D}$ case.

However, there is one more inter-nodal relationship between node 1 and $2, d_{21}$, dashed line, see Figure 2b; and thus, one more inter-nodal relationship can be added to the measurements. Therefore, the matrix A can be rewritten as follows:

$$
A=\left[\begin{array}{ccc}
-I_{1} & 0 & I_{3} \\
0 & -I_{1} & I_{3} \\
-I_{1} & I_{3} & 0
\end{array}\right]
$$

Similarly, if the number of nodes is 4 (5), one can consider 3 (6) inter-nodal distances among the reference nodes and 3 (4) inter-nodal distances between the user nodes and the reference nodes. Naturally, with the increasing number of nodes in the network, more inter-nodal measurements can be obtained and measured. Therefore, the proposed 'Network-based Estimation' method can provide stronger geometry, as compared to a single distance measurement, as well as the redundancy.

Three network-based collaborative navigation algorithms, namely:

(1) RLESS which employs the minimum constraints to overcome the rank deficiency by fixing one of the known positions; 
(2) SCLESS which imposes the stochastic constraints on the unknown position using a priori coordinate variance and

(3) BLIMPBE which minimizes the biases for a subset of parameters (the position) are tested in a network composed of the various kinematic nodes with the objective to compare, evaluate, and, ultimately, to identify the best method for collaborative navigation.

The general measurement (observation) model is shown in Equation (3) and is recalled as:

$$
\vec{y}=A \cdot \vec{x}+\vec{v}, \quad \vec{v} \sim N\left(0, \Sigma=\sigma_{0}^{2} P^{-1}\right)
$$

where:

$\vec{y}$ is the $3 m \times 1$ inter-nodal measurement vector of $\mathrm{x}, \mathrm{y}, \mathrm{z}$ components.

$A$ is the $3 m \times 3 n$ design matrix with rank of $q(q \leqslant n), x$ is $3 n \times 1$ unknown vector of position difference in $\mathrm{x}, \mathrm{y}, \mathrm{z}$.

$\vec{v}$ is the additive noise vector assuming the normal distribution with zero mean and variance-covariance of $\Sigma$.

$\sigma_{0}^{2}$ is the unit-less variance component.

$P$ is the weight matrix of the measurements.

2.1 Restricted Least-Squares Solution (RLESS). In the RLESS, the minimum constraint is given by fixing the best known coordinates in a network. For example, if there are two reference nodes and one user node, one reference node which has the best accuracy (e.g., master node) is fixed for a minimally constrained adjustment. Thus, it could be the best approach if only one node has high accuracy coordinates.

The minimally constrained solutions are obtained using the following constraint equation:

$$
\kappa_{0}=K \cdot \vec{x}
$$

where:

$\kappa_{0}$ is the $l \times 1$ constraint vector.

$K$ is the $l \times m$ corresponding coefficient matrix and $\operatorname{rank}(K)=n-q$.

$q$ is the number of coordinates fixed.

The following estimate and the corresponding variance-covariance matrix for the unknown vector, $\hat{x}_{R L E S S}$, can be obtained using the general least-squares method:

$$
\begin{gathered}
\hat{x}_{R L E S S}=\left(N+K^{T} K\right)^{-1}\left(c+K^{T} \kappa_{0}\right) \\
D\left\{\hat{x}_{R L E S S}\right\}=\sigma_{0}^{2}\left(N+K^{T} K\right)^{-1} N\left(N+K^{T} K\right)^{-1}
\end{gathered}
$$

where $[N c]=A^{T} P[A y]$.

Alternatively, the estimate and corresponding covariance matrix for the RLESS can be obtained using the general recursive least-squares method (see the detailed derivations in Lewis, 1986).

2.2 Stochastically Constrained Least-Squares Solution (SCLESS). If prior information about the nodes is available (e.g., known or best approximation of the 
coordinates of the nodes) with their variance-covariance matrix, the SCLESS method can be used. Similar to Equation (7), the constraints with random errors are formulated as:

$$
z_{0}=K \cdot x+e_{0}, \quad e_{0} \sim\left(0, \Sigma_{0}=\sigma_{0}^{2} P_{0}^{-1}\right)
$$

where:

$z_{0}$ is the $l \times 1$ stochastic constraints vector.

$e_{0}$ is the corresponding random errors with zero mean and variance-covariance of $\Sigma_{0}$.

$P_{0}$ is the positive-definite weight matrix.

The rank of $K$ matrix is $l \geqslant m-q$.

Since the observation vector and the stochastic constraints are uncorrelated, the least-squares estimates, $\hat{x}_{S C-L E S S}$, and the variance-covariance matrix, $D\left\{\hat{x}_{S C-L E S S}\right\}$, of the unknown vectors are:

$$
\begin{gathered}
\hat{x}_{S C-L E S S}=\left(N+K^{T} P_{0} K\right)^{-1}\left(c+K^{T} P_{0} z_{0}\right) \\
D\left\{\hat{x}_{S C-L E S S}\right\}=\sigma_{0}^{2}\left(N+K^{T} P_{0} K\right)^{-1}
\end{gathered}
$$

2.3 Best Linear Minimum Partial Bias Estimation (BLIMPBE). Finally, the BLIMPBE, which was originally developed to minimize the biases at a specific receiver positions in a rank-deficient GPS network adjustment, estimates the unknowns with the minimum biases using the selection matrix (Schaffrin and Iz, 2001; Snow, 2002). For example, if two reference nodes have better accuracy than all other reference nodes (i.e., the number of all nodes is more than three) one can employ only two reference nodes in the BLIMPBE method using a selection matrix. The SCLESS uses all reference nodes in a network, while the BLIMPBE employs only selected reference nodes. Therefore, it could be the best approach if one can find (or select) the best reference nodes among all reference nodes in the network.

The selection matrix, which has the following form, should have sufficient rank to overcome the rank-deficiency in the system.

$$
S_{m \times m}=\left[\begin{array}{cc}
I_{3 \times 3, s} & 0 \\
0 & 0
\end{array}\right]
$$

where $I_{3 \times 3, s}$ is the 3 by 3 identity matrix with the dimension of $s$ according to the number of selected reference nodes from the entire network.

Since the detailed derivations are available in ibid., the resulting estimates, $\hat{x}_{B L I M P B E}$, and the corresponding variance-covariance matrix, $D\left\{\hat{x}_{B L I M P B E}\right\}$, are given at Equations (14) and (15):

$$
\begin{gathered}
\hat{x}_{B L I M P B E}=\left[S N(N S N S N)^{-} N S\right] c \\
D\left\{\hat{x}_{B L I M P B E}\right\}=\sigma_{0}^{2}\left[S N(N S N S N)^{-} N S\right]
\end{gathered}
$$

3. CENTRALiZED KALMAN FilTER. The Centralized Filter (CF) represents globally optimal estimation accuracy for the implemented system models (Knight et al, 1993; Grejner-Brzezinska and Wang, 1998; Carlson, 2002; Zhang et al., 


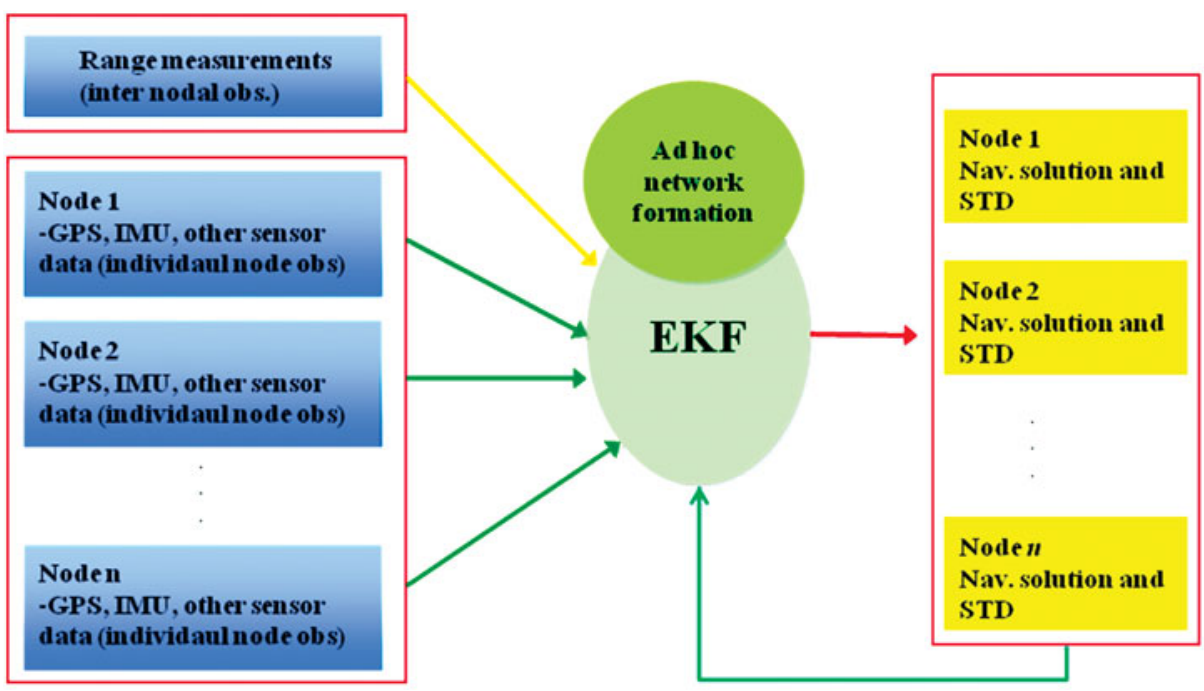

Figure 3. Centralized filter for collaborative navigation.

2002). A CF may lead to significant computation loads, but it serves as an ideal benchmark for other filters. Therefore, in this paper, we implemented a centralized linear Kalman filter and compared its performance with respect to three proposed network-based collaborative navigation algorithms. In Figure 3, all measurements collected at the nodes and the inter-nodal range measurements are processed by a single centralized Kalman filter. Figure 3 illustrates a generic case, when non-linear measurements and/or dynamic model are used, while in the examples presented here a linear Kalman filter is used.

The full derivation of the linear Kalman filter for collaborative navigation is well presented in (Sanderson, 1997). Here, only a summary is given.

A linear measurement model of collaborative navigation can be described as the random variable, $\vec{x}$, which is the vector of nodes position with a priori distribution, $P_{k-1}$, and measurements is given as $\vec{y}$, with covariance matrix, $R_{v}$. Based on Gaussian probability density function the linear measurement model can be solved as the minimum mean-square error estimation of the Kalman filter.

A linear estimator, $\hat{\vec{x}}$, for nodes positions, $\vec{x}_{k}$, is given as

$$
\hat{\vec{x}}_{k}=\hat{\vec{x}}_{k-1}+P_{k} H_{k}^{T} R_{v}^{-1}\left(\vec{y}_{k}-H_{k} \hat{\vec{x}}_{k-1}\right)
$$

where:

matrix $H_{k}$ shows the relationship between states and inter-nodal range measurements.

$R_{v}$ is the covariance matrix of the inter-nodal range measurement errors.

The covariance of the estimates is given as:

$$
P_{k}=\left(P_{k-1}^{-1}+H_{k}^{T} R_{v}^{-1} H_{k}\right)^{-1}
$$




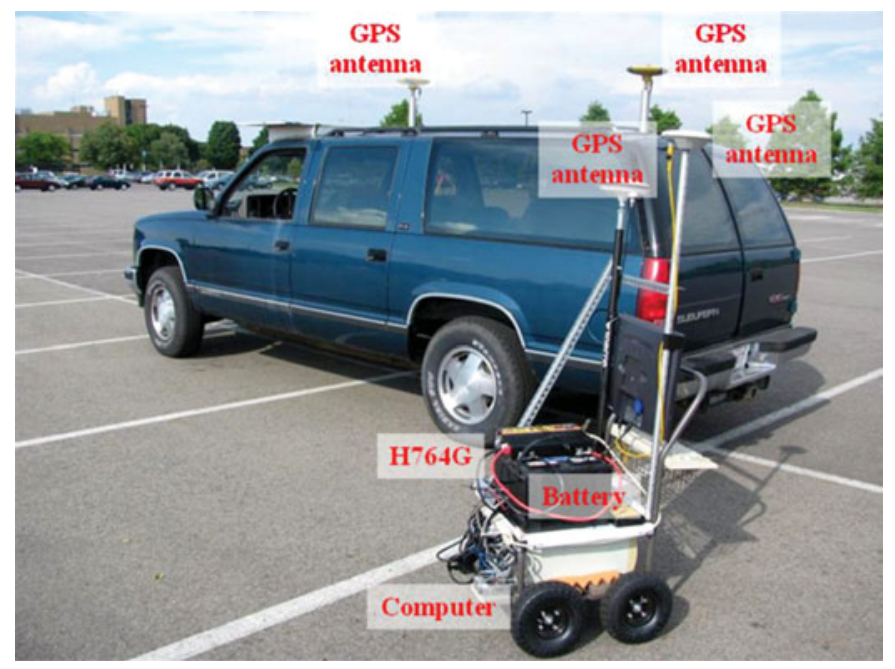

Figure 4. 'GPSVan' and 'mobile cart'.

4. FIELD TEST AND SIMULATION. To evaluate the network-based collaborative navigation algorithms, a field test was performed on 11 August. 2009 on West Campus at The Ohio State University. The three GPS stations and two mobile platforms (referred to as 'GPSVan' and 'mobile cart'), were used in the test. In the GPSVan, there are two navigation-grade IMUs and two geodetic-grade GPS receivers, while the mobile cart carries a tactical-grade IMU, two GPS receivers and a 12 volt battery (Figure 4). The GPSVan and the cart were moved along the varyingdynamic trajectories around the three GPS base stations (Figure 5).

To process the kinematic GPS data, the AIMS-Pro software ver. 2.0, developed at the Satellite Positioning and Inertial Navigation (SPIN) Laboratory at OSU, was employed. The position fixes were provided at the data-sampling rate of $1 \mathrm{~Hz}$. The distances to the base stations were rather short (up to $200 \mathrm{~m}$ ) for the entire experiment. Thus, L1 carrier phase measurements, after removing fixed doubledifferential integer ambiguity, were used to generate the reference solutions for all network nodes.

In order to generate a 5-node kinematic network, the GPSVan trajectory was used as a (virtual) reference station and four reference nodes (HL, N2, N3 and N4 in Figure 5) and four different trajectories were generated. Finally, the trajectory of the mobile cart (C2, in Figure 5) was assigned as user node. Based on the pre-generated trajectories, the four 3-dimensional ranges in ECEF X, Y, Z (i.e., position difference or baseline components) between reference nodes and the user node and the six ranges between the reference nodes (see Figure 2d) were computed at all epochs along with their variance-covariance information. This was done to simulate the inter-nodal 3-dimensional distance measurements, as no actual 3-dimensional measurements were acquired (it is very difficult to obtain the 3-dimensional range measurements in practice). Next, simulated errors were added to the $\mathrm{C} 2$ node; the initial coordinate uncertainty of $5 \mathrm{~m}$ was added to $\mathrm{x}, \mathrm{y}$, and $\mathrm{z}$ components as biases, and random error, ranging between $-50 \mathrm{~cm}$ and $+50 \mathrm{~cm}$ was also applied. Next, the positioning solutions of the network-based collaborative navigation were computed and compared with 


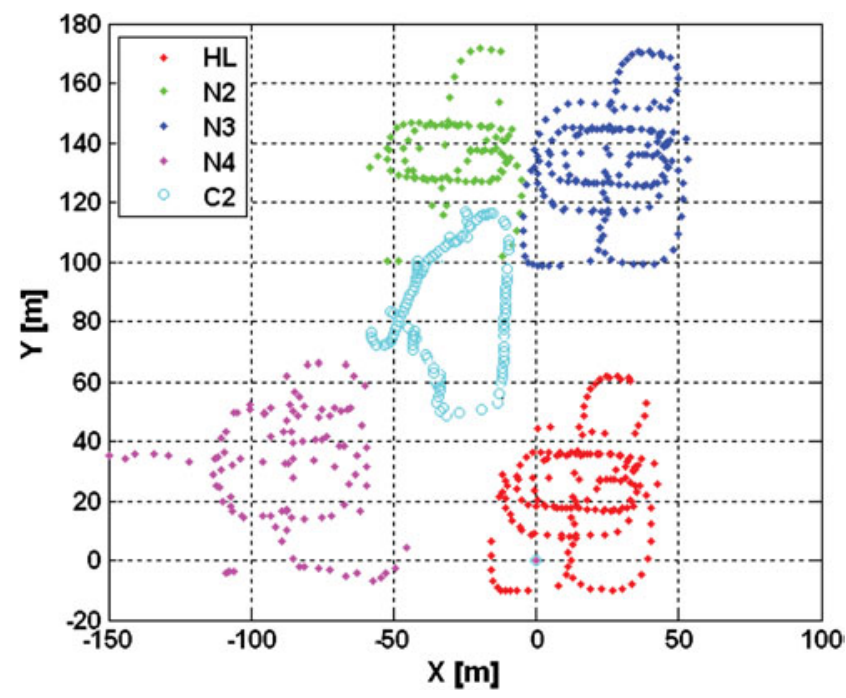

Figure 5. The simulated trajectory of the five nodes.

each original user node position (i.e., the reference carrier-phase-based GPS solutions using AIMS-Pro).

5. SIMULATED RESULTS AND ANALYSIS. In 'Scenario 1' (3-node case), there is one range measurement between the reference nodes and two range measurements between the user and the reference nodes. In 'Scenario 2' (5-node case), the six ranges between the reference nodes and the four ranges between the user node and the reference nodes are used to obtain the position estimates of the user node $(\mathrm{C} 2$ in figure 5). Figures 6 and 7 show the position difference between the simulated user trajectory with biases and random errors added, and the estimated user positions for the four network-based collaborative algorithms: the SCLESS, the BLIMPBE, the RLESS, and the Kalman filter for two cases (Scenarios 1 and 2) where the number of nodes equal three and five, respectively.

5.1. Scenario 1: 3-Nodes in a Network. The added biases and random error in the user node's coordinates were significantly decreased from the simulated erratic coordinates, through the three network-based collaborative navigation algorithms, especially by the SCLESS, the BLIMPBE, the RLESS and the Kalman filter (see Figure 6). The SCLESS and BLIMPBE method showed better performance (especially in the standard deviation) than the RLESS and the Kalman filter. The RLESS and the Kalman filter showed the comparable biases, but the random errors were not adequately removed, as compared to the two other methods. The SCLESS and BLIMPBE solutions were almost the same for the user node, while the SCLESS solution absorbed the (added) biases into the network. Therefore, the estimated coordinates of user node in a network by using the a priori variance information were affected accordingly. Because RLESS is predominantly dependent on the accuracy of the coordinates to be fixed, it is not recommended for use in the network-based estimation. Table 1 shows the statistical results of the position difference between the 

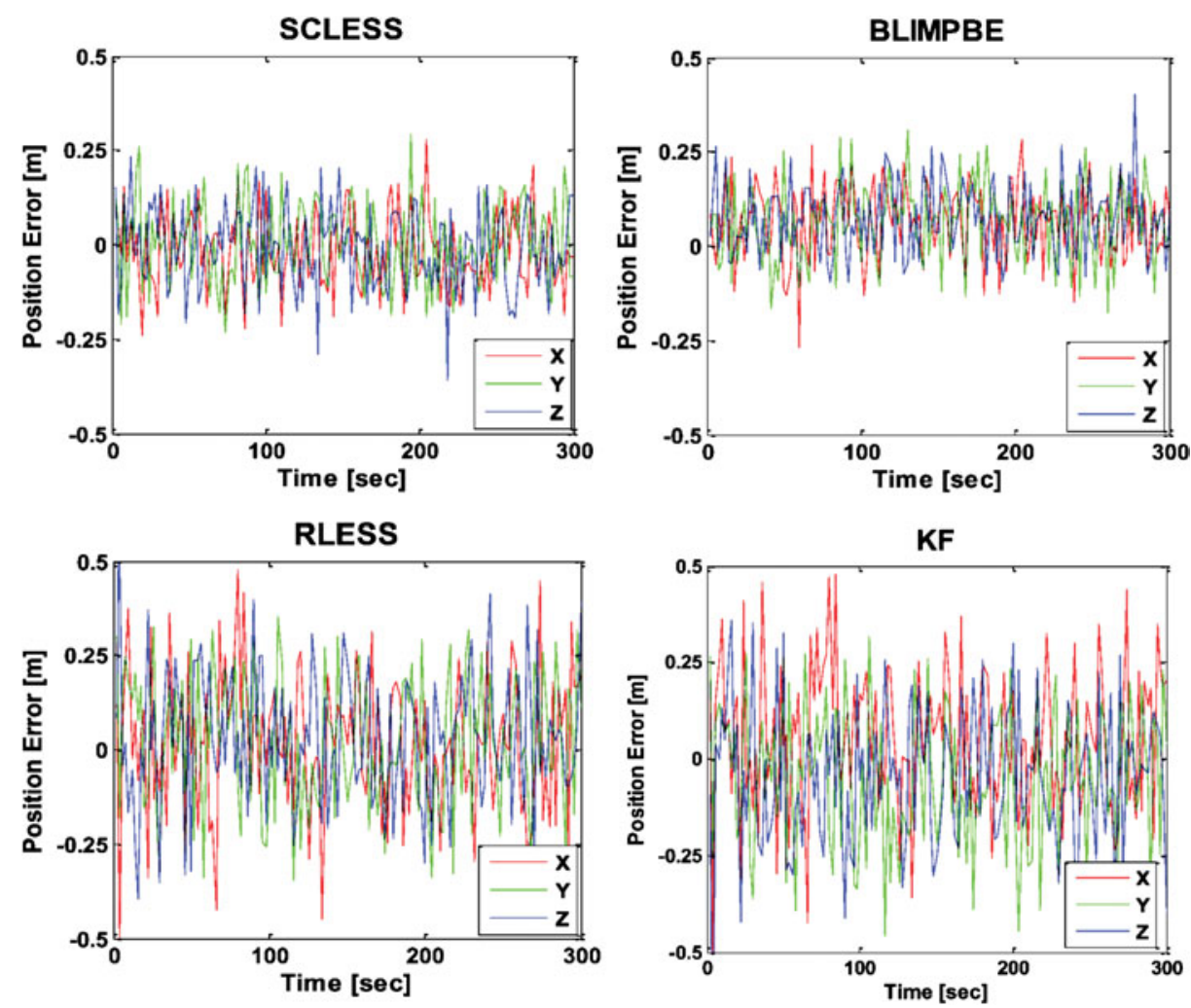

Figure 6. Position error of the user node (C2) according to three network-based collaborative algorithms and Kalman Filter (KF) (3-node case, in Figure 2b).

original reference solutions and the estimated coordinates from the network-based algorithm, using the simulated biased data with random errors, as explained earlier.

5.2. Scenario 2: 5-Node Network. As can be seen in Figure 7 and Table 2 (statistical position error result of the user node C2), the SCLESS, the BLIMPBE, and the RLESS method performed better than the Kalman filter method because the increased number of reference nodes (i.e., inter-nodal distance measurements) can provide stronger geometry and redundancy of the proposed network-based collaborative navigation algorithms. The SCLESS method showed best standard deviations (also, in the Root Mean Square [RMS]) of the user node among all proposed estimation methods. Similarly to Scenario 1, the RLESS showed worse accuracy of the user nodes than that of other two network-based collaborative navigation algorithms.

Overall, it can be seen that the proposed network-based collaborative algorithm methods can enhance the accuracy and the stability in the coordinate estimation of a user node by using only inter-nodal distance measurements when a GPS signal is not available. The methods and analysis presented in this paper provide a way to improve the solutions by simply building a network and applying these statistical collaborative navigation estimation methods. Although only inter-nodal range measurement is used in this study, additional ranging solutions and multi-sensor output could be applied to 
NO. 3 NETWORK-BASED COLLABORATIVE NAVIGATION IN GPS-DENIED 455

Table 1. The statistical result of user node $\mathrm{C} 2$ of three network-based collaborative navigation algorithms and Kalman Filter (KF) solutions (3-node case).

\begin{tabular}{lcccc}
\hline Algorithm & {$[\mathrm{cm}]$} & Mean & Std. Dev. & RMS \\
\hline \multirow{4}{*}{ SCLESS } & $\mathrm{X}$ & $0 \cdot 037$ & $0 \cdot 099$ & $0 \cdot 106$ \\
& $\mathrm{Y}$ & $0 \cdot 057$ & $0 \cdot 111$ & $0 \cdot 124$ \\
& $\mathrm{Z}$ & $0 \cdot 040$ & $0 \cdot 110$ & $0 \cdot 117$ \\
BLIMPBE & Total & & $0 \cdot 201$ & $0 \cdot 116$ \\
& $\mathrm{X}$ & $0 \cdot 064$ & $0 \cdot 097$ & $0 \cdot 119$ \\
& $\mathrm{Y}$ & $0 \cdot 063$ & $0 \cdot 101$ & $0 \cdot 122$ \\
& $\mathrm{Z}$ & $0 \cdot 076$ & $0 \cdot 095$ & \\
RLESS & Total & & $0 \cdot 206$ & $0 \cdot 180$ \\
& $\mathrm{X}$ & $0 \cdot 030$ & $0 \cdot 178$ & $0 \cdot 175$ \\
& $\mathrm{Y}$ & $0 \cdot 035$ & $0 \cdot 172$ & $0 \cdot 189$ \\
& $\mathrm{Z}$ & $0 \cdot 044$ & $0 \cdot 184$ & \\
KF & Total & & $0 \cdot 319$ & $0 \cdot 174$ \\
& $\mathrm{X}$ & $0 \cdot 024$ & $0 \cdot 173$ & $0 \cdot 182$ \\
& $\mathrm{Y}$ & $-0 \cdot 040$ & $0 \cdot 178$ & $0 \cdot 175$ \\
& $\mathrm{Z}$ & $-0 \cdot 027$ & $0 \cdot 173$ & $0 \cdot 303$ \\
\hline
\end{tabular}
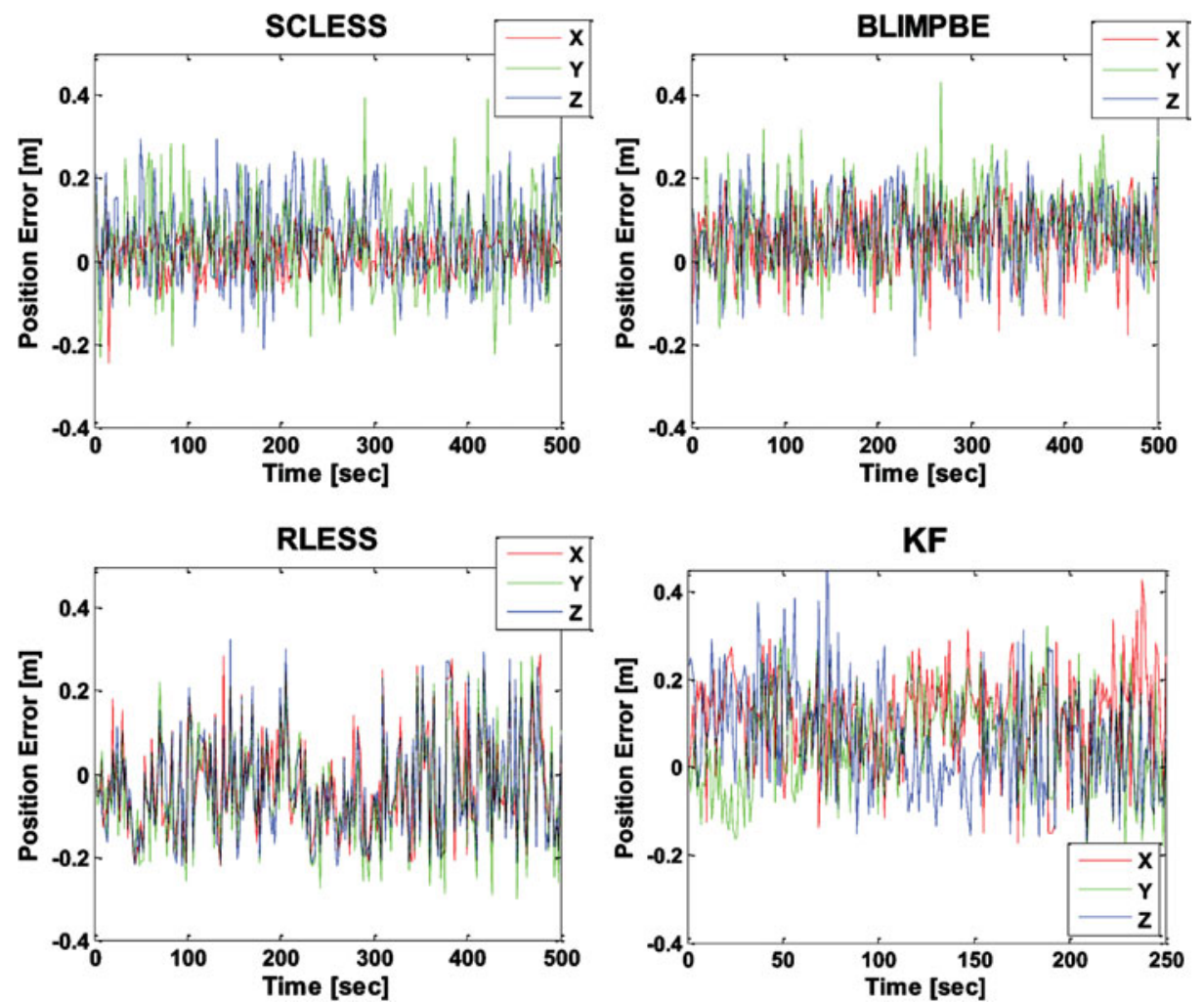

Figure 7. Position error of the user node (C2) according to three network-based collaborative algorithms and the Kalman Filter (KF) (5-node case, in Figure 2d). 
Table 2. The statistical results of fifth user node $\mathrm{C} 2$ of three network-based collaborative navigation algorithms and Kalman Filter (KF) solutions (5-node network).

\begin{tabular}{lcccc}
\hline Algorithm & {$[\mathrm{cm}]$} & Mean & Std. Dev. & RMS \\
\hline \multirow{4}{*}{ SCLESS } & $\mathrm{X}$ & $-0 \cdot 013$ & $0 \cdot 072$ & $0 \cdot 073$ \\
& $\mathrm{Y}$ & $0 \cdot 006$ & $0 \cdot 101$ & $0 \cdot 101$ \\
& $\mathrm{Z}$ & $0 \cdot 011$ & $0 \cdot 100$ & $0 \cdot 100$ \\
BLIMPBE & Total & & & $0 \cdot 162$ \\
& $\mathrm{X}$ & $-0 \cdot 012$ & $0 \cdot 084$ & $0 \cdot 085$ \\
& $\mathrm{Y}$ & $0 \cdot 021$ & $0 \cdot 100$ & $0 \cdot 102$ \\
& $\mathrm{Z}$ & $0 \cdot 013$ & $0 \cdot 109$ & $0 \cdot 110$ \\
RLESS & Total & & & $0 \cdot 172$ \\
& $\mathrm{X}$ & $-0 \cdot 050$ & $0 \cdot 095$ & $0 \cdot 099$ \\
& $\mathrm{Y}$ & $-0 \cdot 041$ & $0 \cdot 109$ & $0 \cdot 117$ \\
& $\mathrm{Z}$ & $-0 \cdot 042$ & $0 \cdot 108$ & $0 \cdot 117$ \\
KF & Total & & & $0 \cdot 205$ \\
& $\mathrm{X}$ & $0 \cdot 112$ & $0 \cdot 108$ & $0 \cdot 156$ \\
& $\mathrm{Y}$ & $0 \cdot 052$ & $0 \cdot 113$ & $0 \cdot 125$ \\
& $\mathrm{Z}$ & $0 \cdot 029$ & $0 \cdot 102$ & $0 \cdot 106$ \\
& Total & & & $0 \cdot 227$ \\
\hline
\end{tabular}

the network-based collaborative navigation case as well. The position errors RMS of SCLESS (BLIMPBE) were smaller by about 33\% (32\%) with respect to the Kalman filter results in Scenario 1. In Scenario 2, SCLESS, BLIMPBE and RLESS position errors were smaller that that of the Kalman filter by about $28 \%, 24 \%$, and $9 \%$, respectively. Even though the accuracy improvement achieved by using one of the statistical-based methods is at sub-decimetre level, which may not be relevant in land vehicle navigation, it has substantial relevance in navigating small platforms, such as swarms of autonomous miniature vehicles or robots.

6. CONCLUSIONS. It was demonstrated that the proposed statistical network-based 'Collaborative Navigation' algorithms can improve the position accuracy of the user node in the network based on field test data and simulated ranges. The 'GPSVan' and the 'mobile cart' with three geodetic GPS base stations were used in the field tests discussed here. Three network-based collaborative navigation algorithms, namely the Stochastic Constrained Least-Squares Solution (SCLESS), the Best Linear Minimum Partial Bias Estimation (BLIMPBE) and the Restricted Least-Squares Solution (RLESS) were proposed and compared to the Kalman filter to obtain accurate position of the user node and all other nodes in a network under realistic field conditions. The SCLESS method showed the best performance, especially, in the standard deviation, among the proposed 'Network-based Estimation' methods and the Kalman filter. In other proposed network-based estimation methods, the overall performance of the SCLESS and the BLIMPBE was better than that of the RLESS in the 5-node and 3-node cases. Therefore, it is concluded that the statistical collaborative navigation algorithm is a preferred estimation method for the collaborative navigation applications such as robots, ground-users and autonomous miniature vehicles. 


\section{REFERENCES}

Berefelt, F., Boberg, B., Nygårds, J., Strömbäck, P. and Wirkander, S.L. (2004). Collaborative GPS/INS Navigation in Urban Environment. Proceedings of the 2004 National Technical Meeting of The Institute of Navigation, San Diego, CA.

Brown, A. and Nordlie, J. (2006). Integrated GPS/TOA navigation using a positioning and communication software defined radio. Proceeding of PLANS 2006, San Diego, CA.

Carlson, N. (2002). Federated Filter for Distributed Navigation and Tracking Application. Proceedings of the ION 58th Annual Meeting, Albuquerque, NM.

Fox, D., Burgard, W., Kruppa, H. and Thrun, S. (1999). Collaborative Multi-Robot Localization. Proceedings of the 23rd German Conference on Artificial Intelligence, Springer Verlag.

Grejner-Brzezinska, D.A. and Wang, J. (1998). Gravity Modeling for High-Accuracy GPS/INS Integration, Navigation, 45, 209-220.

Grejner-Brzezinska, D. A., Toth, C. K., Sun, H., Wang, X. and Rizos, C. (2008). GPS/INS/PL/TLS Integration Supporting Navigation of Geophysical Sensors for Unexploded Ordnance Detection and Discrimination. Proceedings of 13th FIG International Symposium on Deformation Measurements and Analysis, 4th IAG Symposium on Geodesy for Geotechnical and Structural Engineering, Lisbon, Portugal.

Grejner-Brzezinska, D.A., Toth, C.K., Li, L., Park, J., Wang, X., Sun, H., Gupta, I.J., Huggins, K. and Zheng, Y.F. (2009). Positioning in GPS-challenged Environments: Dynamic Sensor Network with Distributed GPS Aperture and Inter-nodal Ranging Signals. Proceedings of the 22nd International Technical Meeting of The Satellite Division of the Institute of Navigation (ION GNSS 2009), Savannah, GA.

Kaba, J. and Wu, S. (2009). Distributed Multi-Sensor Fusion for improved Collaborative GPS-denied Navigation. Presentation at Joint Navigation Conference.

Knight, D.T., Osborne, A., Snow, R. and Kim, D. (1993). Demonstration of a New Tightly-Coupled GPS/ INS. Proceedings of 6th International Technical Meeting, ION, Salt Lake City, Utah.

Lee, J.K. and Jekeli, C. (2011). Rao-Blackwellized Unscented Particle Filter for a Handheld Unexploded Ordnance Geolocation System using IMU/GPS. The Journal of Navigation, 64, 327-340.

Lewis, F. L. (1986). Optimal Estimation. Wiley, New York.

Mirabadi, A., Schmid, F. and Mort, N. (2003). Multisensor Integration Methods in the Development of a Fault-Tolerant Train Navigation System. The Journal of Navigation, 56, 385-398.

Panzieri, S., Pascucci, F. and Setola, R. (2005). Interlaced Extended Kalman Filter for Real Time Navigation. Intelligent Robots and Systems, IEEE/RSJ International Conference on Digital Object Identifier: 10.1109/IROS.2005.1544979.

Sanderson, A. C. (1997). A Distributed Algorithm for Cooperative Navigation Among Multiple Mobile Robots. Advanced Robotics, 12, 335-349.

Schaffrin, B. and Iz, H.B. (2001). BLIMPBE and its Geodetic Applications. Vistas for Geodesy in the New Millennium, Adam and Schwarz. Springer, Berlin.

Sharaf, R. and Noureldin, A. (2007). Sensor Integration for Satellite-Based Vehicular Navigation Using Neural Networks. Neural Networks, IEEE Transactions on, 18, 589-594.

Snow, K. B. (2002). Applications of Parameter Estimation and Hypothesis Testing to GPS Network Adjustments. Ohio State University Technical Report No. 456, xii +107.

Zhang, H., Lennox, B., Goulding, P., and Wang, Y. (2002). Adaptive Information Sharing Factors in Federated Kalman Filtering. Proceedings of IFAC 15th Triennial World Congress, Barcelona, Spain. 